\title{
A Case Series of Esophageal Lichen Planus: An Underdiagnosed Cause of Dysphagia
}

\author{
Puja Rai ${ }^{1}$, Mahmoud Y Madi ${ }^{1}$, Raymond Lee ${ }^{2}$, Aaron Dickstein ${ }^{2}$ \\ Departments of Internal Medicine ${ }^{1}$ and Gastroenterology ${ }^{2}$, Tufts Medical Center, Boston, MA, USA
}

Lichen planus (LP) is a chronic mucocutaneous inflammatory condition that typically affects middle-aged adults. Esophageal involvement in LP is rare and underrecognized, often leading to delayed diagnosis and treatment of LP. Herein, we describe three cases of esophageal LP (ELP) in clinically symptomatic patients with endoscopic lesions in the upper to mid-esophagus. This case series suggests that ELP is be more common than was previously thought and emphasizes that clinicians should have a high index of suspicion for this diagnosis, particularly when evaluating proximal esophageal lesions in patients presenting with dysphagia. The series also highlights the successful treatment of our patients with budesonide-honey slurry. (Korean J Helicobacter Up Gastrointest Res 2019;19:266-271)

Key Words: Lichenoid eruptions; Lichen planus; Skin diseases, papulosquamous

\section{INTRODUCTION}

Lichen planus (LP) is an idiopathic, inflammatory disease that typically affects middle-aged adults with clinical manifestations in the skin, scalp, nails, and mucous membranes. ${ }^{1}$ Esophageal involvement in LP is underrecognized, with approximately 80 published cases in medical literature since 1982. ${ }^{2}$ Endoscopic findings may be subtle, and histology may be inconclusive. The diagnosis is therefore difficult and typically delayed. Currently, there are no standardized management guidelines but use of steroids or immunomodulating agents in conjunction with endoscopic dilations have shown positive effects.

Informed consent was obtained from the patients for publication of their cases.

\section{CASE REPORT}

\section{Case 1}

A 69-year-old man with a past medical history of hyper-

Received: June 5, 2019 Revised: July 12, 2019 Accepted: July 12, 2019

Corresponding author: Mahmoud Y Madi

Department of Internal Medicine, Tufts Medical Center 800 Washington Street, Boston, MA 02111, USA

Tel: +1-857-400-6473, Fax: +1-617-636-7119, E-mail: Mahmood_madi@hotmail.com tension and colonic polyps presented with subacute, episodic dysphagia of 4 months duration. He reported six episodes of dysphagia to solids, all occurring after eating. His symptoms would self-resolve within 3 hours. He noted intentional $10 \mathrm{~kg}$ weight loss over the past few months and had no other associated symptoms. Physical exam of the abdomen was unremarkable, and he had no evidence of any oral or skin lesions. Medication list included lisinopril and low-dose aspirin. Barium swallow was notable for smooth

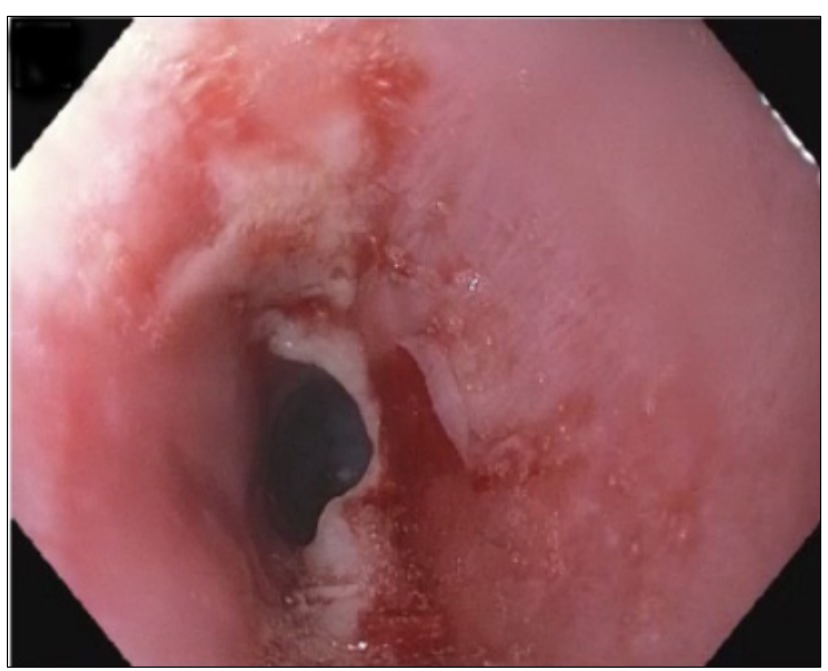

Fig. 1. Endoscopy showing significant stenosis with ulceration in the upper esophagus at $20 \mathrm{~cm}$, which was dilated to $10 \mathrm{~mm}$ using a Savary dilator.

Copyright $\odot 2019$ Korean College of Helicobacter and Upper Gastrointestinal Research

(a) The Korean Journal of Helicobacter and Upper Gastrointestinal Research is an Open-Access Journal. All articles are distributed under the terms of the Creative Commons Attribution Non-Commercial License (http:// creativecommons.org/licenses/by-nc/4.0) which permits unrestricted non-commercial use, distribution, and reproduction in any medium, provided the original work is properly cited. 
narrowing of upper esophagus without masses or rings and non-passage of a $13 \mathrm{~mm}$ tablet. Endoscopy revealed friable mucosa and concentric rings with luminal narrowing in the esophagus without overt stenosis extending between 20 30 cm from the incisors with biopsy results showing inflammation without increased eosinophils. Despite adhering to a soft diet with small bites and omeprazole $20 \mathrm{mg}$ twice daily, patient continued to have intermittent dysphagia to solids approximately twice monthly. Chest CT showed no focal abnormality or external compression of the esophagus. Repeat endoscopy performed more than 1 year later showed significant stenosis with ulceration in the upper esophagus at $20 \mathrm{~cm}$, which was dilated to $10 \mathrm{~mm}$ (Fig. 1). He had a subsequent endoscopy with repeat dilatation using a Savary

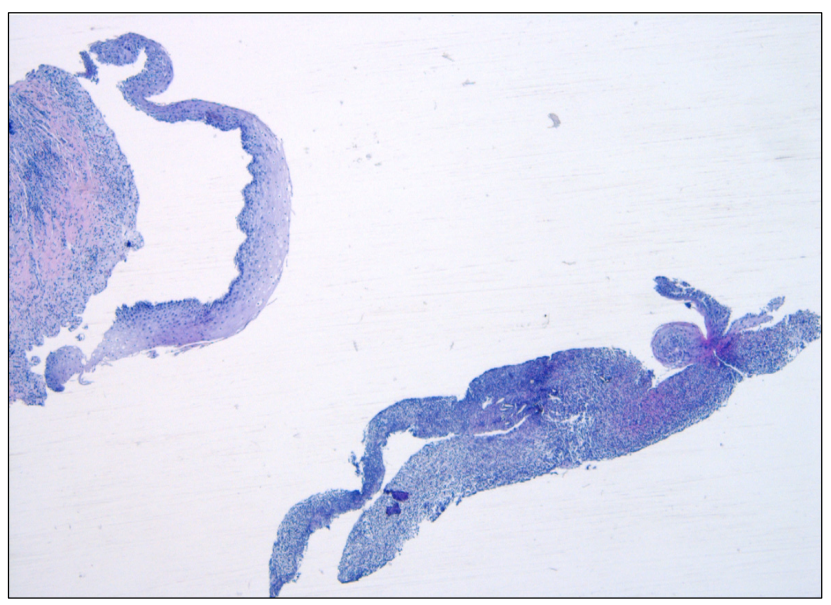

Fig. 2. Biopsy specimen revealing a prominent neutrophilic ulcer and patchy lymphoid infiltrate in the submucosa and focal subepithelial clefting. The lymphoid infiltrate has a hint of band-like distribution. No colloid bodies are identified. Findings of subepithelial clefting and lymphoid infiltrate raise a suspicion of lichen planus (H\&E, $\times 40)$. bougie (Cook Medical, Bloomington, IN, USA) with a guidewire. The largest dilator measured $10 \mathrm{~mm}$. Repeat biopsy showed squamous intraepithelial lymphocytosis with focal subepithelial clefting concerning for LP (Fig. 2). Hepatitis $\mathrm{C}$ antibody testing was negative. He was started on swallowed budesonide $2 \mathrm{mg}$ twice daily mixed with 8 $\mathrm{mL}$ honey which was eventually tapered. Patient clinically improved with steroid therapy and repeated interval dilations with subsequent endoscopic evidence of near resolution of mucosal friability and overt signs of inflammation.

\section{Case 2}

A 79-year-old woman with a past medical history of oral and genital LP, and breast cancer status-post chest radiation therapy presented with episodes of sharp, fleeting mid-chest discomfort of 6 months duration. Symptoms occurred while eating or drinking, lasting 30 seconds and then resolving. She also reported associated heartburn but denied dysphagia. Initial proton pump inhibitor (PPI) therapy and antacids did not provide relief. The rest of her home medication list included aspirin, estradiol cream and methenamine. Physical exam was notable for known murmur at cardiac apex and normal abdominal exam. She was initially thought to have possible esophageal spasm or Schatzki rings, but barium swallow did not show any obstruction or corkscrewing. There was evidence of moderate gastroesophageal reflux disease (GERD) and multiple hang-ups of barium tablet with occasional extra contractions. Labs revealed normal thyroid stimulating hormone (TSH) and negative Helicobacter pylori (H. pylori) antibody. She was re-trialed on high dose PPI
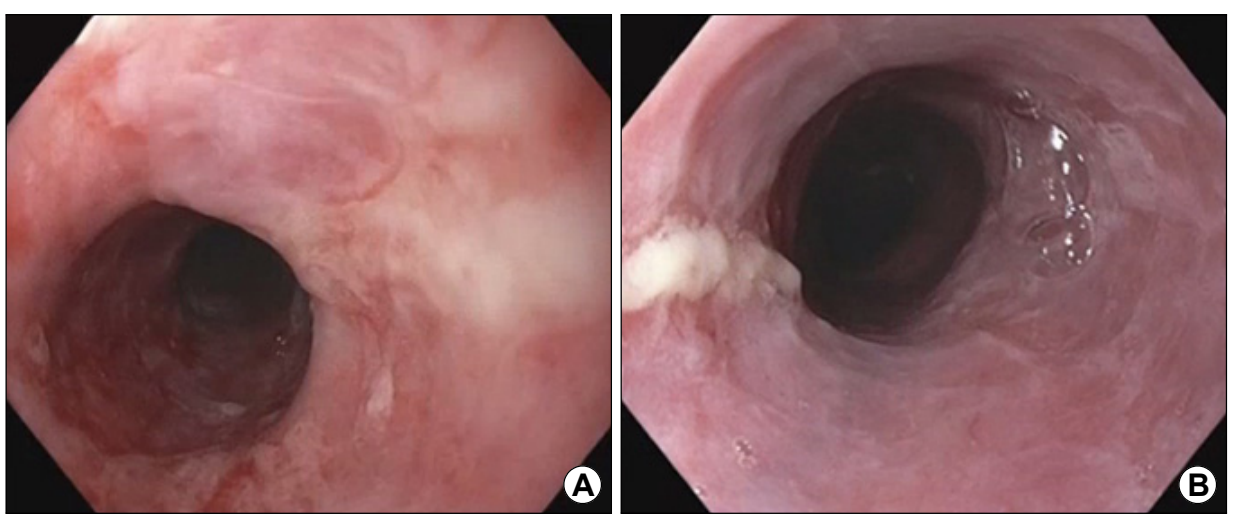

Fig. 3. Endoscopic evidence of severe esophagitis throughout the esophagus, including mucosal friability and ulceration with sloughing and erosions and mild trachealization of the upper and mid esophagus. (A) Upper esophagus, (B) mid-esophagus. 
therapy which initially improved symptoms, but she soon developed recurrence of symptoms. Repeat endoscopy showed severe esophagitis along the entire length of the esophagus, including mucosal friability and ulceration with sloughing and erosions, mild trachealization of the upper and mid-esophagus (Fig. 3), and a sliding type hiatal hernia. Biopsy of the upper esophagus revealed squamous mucosa with lymphocytic infiltrate, acute inflammation, apoptotic Civatte bodies and intercellular edema (Fig. 4). Grocott's methenamine silver stain and herpes simplex virus (HSV) stain were negative. Hepatitis $\mathrm{C}$ antibody testing was negative. Esophageal injury related to her history of radiation was considered. However, given her known history of LP of the vagina, vulva, and oral cavity first diagnosed over 30 years ago, possible esophageal involvement was considered, and patient was empirically started on swallowed budesonide 3 mg twice daily mixed with honey. Her symptoms gradually improved on this regimen. Repeat endoscopy about 6 months later showed significantly improved inflammation (Fig. 5).

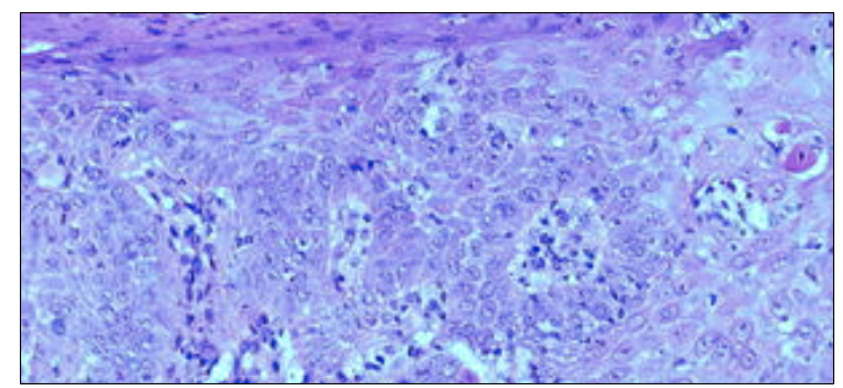

Fig. 4. Evident leukocytic infiltration of the squamous epithelium of the esophagus, with detection of Civatte bodies. These are eosinophilic hyaline spheric bodies resulting from the apoptosis of individual basal cells $(\mathrm{H} \& \mathrm{E}, \times 100)$.
Given her considerable clinical and endoscopic response to budesonide therapy, a diagnosis of esophageal LP (ELP) was confirmed.

\section{Case 3}

A 65-year-old woman with no past medical history presented for initial evaluation of progressive dysphagia and odynophagia to solids over the past 3 years. She noted that since a young age, she had been brushing her teeth with hydrogen peroxide. Barium swallow revealed a long and severe stricture in the upper to mid-esophagus. Although the etiology was initially unclear, it was thought to possibly be from caustic injury from years of hydrogen peroxide use. Physical exam was unremarkable. Labs were normal including TSH and negative hepatitis C screening. She was started on lansoprazole $30 \mathrm{mg}$ daily for reflux. Initial endoscopy showed long smooth stenosis of the esophagus with a ring-like appearance and mucosal sloughing 17 32 cm from the incisors. Lower esophagus was erythematous but not stenotic. Biopsy showed severe active esophagitis with intraepithelial eosinophils, neutrophils and lymphocytes in the proximal and mid esophagus. High-magnification images revealed prominent basal lymphocytic infiltration with multiple Civatte bodies, most consistent with ELP (Fig. 6). The mid-esophagus also showed ulcerative disease with abundant fragments of fibrinopurulent debris. Stains for $H$. pylori, fungi, HSV, and cytomegalovirus were negative. There was no evidence of dysplasia. Symptoms briefly improved with lansoprazole but recurred in few months. Concurrently, the patient developed oral blisters, concerning for a possible au-
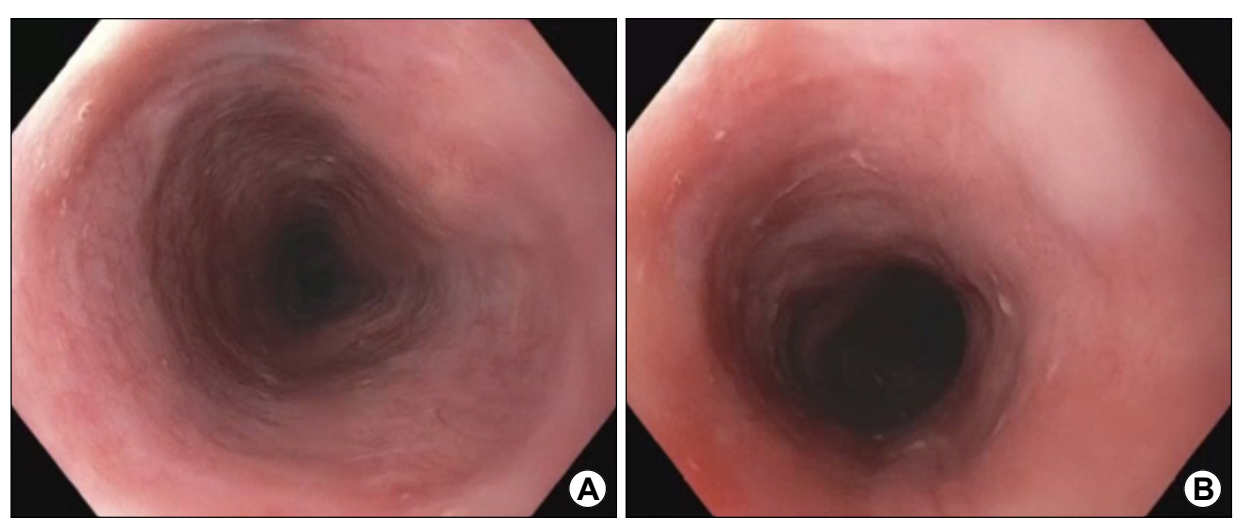

Fig. 5. (A) Upper and (B) mid- esophagus demonstrate significant improvement in inflammation and near resolution of previously noted abnormalities. 


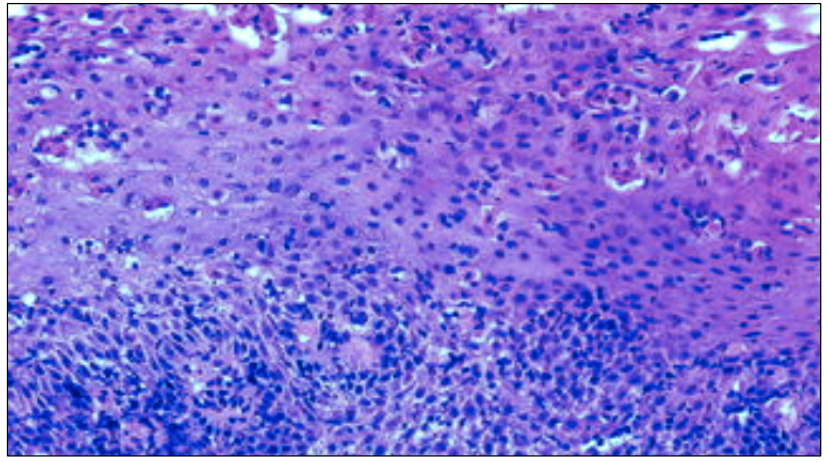

Fig. 6. Predominant lymphocytic infiltration in the basal layer, with multiple Civatte bodies $(\mathrm{H} \& \mathrm{E}, \times 100)$.

toimmune condition. Repeat endoscopy showed persistent stenosis of the mid-esophagus with concentric rings, mucosal friability, and large ulceration with white exudate (Fig. 7). Dilation was not performed due to active ulceration. Ig stains were negative and immunofluorescence studies for IgG and complement component 3 were also negative. Her diagnosis remained uncertain with differential including caustic ingestion or GERD. Given her oral lesions and biopsy revision the diagnosis of ELP was considered. The diagnostic utility of her prior esophageal biopsies was limited given the severity of inflammation. Interestingly, biopsy results of her oral lesions confirmed diagnosis of LP. Therefore, despite inconclusive clinical course, historical and endoscopic findings were consistent with ELP rather than corrosive esophagitis. She was empirically started on swallowed budesonide $3 \mathrm{mg}$ twice daily with honey in addition to PPI. She unfortunately failed to clinically respond with recurrent dysphagia and odynophagia. Serial dilations were attempted using Savary dilators (Cook Medical) $6 \sim 9 \mathrm{~mm}$, and up to $11 \mathrm{~mm}$ on repeat endoscopy and dilation. The patient's symptoms persisted for which she trialed on prednisone, and mycophenolate mofetil which led to gradual improvement in her clinical symptoms. Her disease was deemed refractory to medical and endoscopic therapy and a percutaneous gastrostomy tube was placed for feeding.

\section{DISCUSSION}

ELP is a rare mucosal manifestation of LP but should be considered in patients presenting with dysphagia or odynophagia, especially those with a proximal esophageal

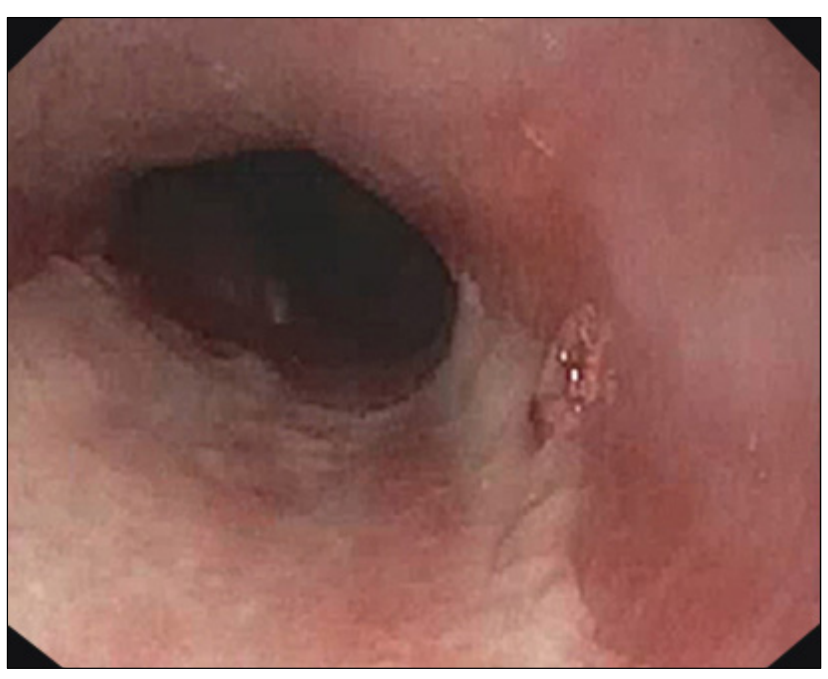

Fig. 7. Persistent stenosis of the mid-esophagus with concentric rings, mucosal friability, and large ulceration with white exudate.

stricture. LP is common as a skin disease, affecting 0.5\% to $2 \%$ of the population. It usually affects middle-aged adults of both genders with equal frequency. ${ }^{3,4}$ Oral LP has an approximate prevalence of $1 \sim 3 \%$ with female predominance, and has strong association with ELP, as demonstrated in our two female patients with oral LP. In fact, ELP can be found in more than $50 \%$ of patients with proven mucocutaneous LP. ${ }^{5}$

Dysphagia and odynophagia are the most common presenting symptoms. ${ }^{6}$ Some patients may have a history of LP while others may present with ELP as their initial manifestation. A review of 27 ELP cases, esophageal disease was the presenting site of LP in 13 patients $(48 \%)^{6}{ }^{6}$ The absence of extraesophageal findings likely contributes to the long-standing duration of symptoms before LP is considered in these patients.

The pathogenesis of LP is unknown but is thought to be from T-cell mediated injury along with inflammatory cytokines. There may also be an association with HCV infection, though this has not yet been well demonstrated. Drug mediated reactions, including with angiotensin-converting enzyme inhibitors, beta blockers, thiazides and anti-malarial drugs may also be implicated. ${ }^{7}$

Depending on their location, strictures may have a variety of causes including reflux disease, mediastinal radiation, caustic ingestion, drug-induced injury, eosinophilic esophagitis, esophageal webs and other skin conditions 
involving the esophagus such as bullous pemphigoid. Patients' symptoms are often mistakenly attributed to GERD. In a review of 27 ELP cases, most of the patients had received multiple dilations and reflux treatments prior to diagnosis. ${ }^{6}$ Notably, ELP can be distinguished from GERD by sparing of the gastroesophageal junction, and lack of clinical response to PPIs. Pill-induced esophagitis may present similarly as ELP with erosive changes, ulcerations and mucosal inflammation. However, changes in pill-induced esophagitis are typically circumscribed. ${ }^{8} \mathrm{~A}$ rare entity with similar appearance to ELP is esophagitis dissecans, which can cause sloughing of large fragments of esophageal mucosal lining. However, esophageal mucosa is extremely fragile with potential Nikolsky's sign: stripping of normal appearing mucosa on withdrawal of biopsy forceps. ${ }^{8,9}$ Medications such as nonsteroidal anti-inflammatory drugs have been implicated in the disease though the exact pathogenesis is unknown.

Combination of clinical symptoms, endoscopic, and histologic findings are often needed to diagnose ELP. On endoscopic evaluation, majority of cases eventually involve stricturing disease in the proximal esophagus, but location may vary. ${ }^{10,11}$ Macroscopic findings are nonspecific but include strictures or webs, stenosis, friable and inflamed mucosa, white discoloration, mucosal thickening, sloughing, superficial ulceration, erosion and subtle ring appearance. Changes may be progressive as those without a stricture present on initial evaluation often developed one later in the course. When ELP is suspected endoscopically, biopsy should be performed. Typical histologic features include band-like lymphocytic infiltrate involving the superficial lamina propria and basal epithelium with presence of necrotic keratinocytes, termed Civatte bodies. However, histopathology can be non-diagnostic in up to $50 \%$ of cases. Therefore, as seen in one of our cases, absence of pathologic confirmation should not exclude the diagnosis.

There are no current standardized guidelines for treatment of ELP. Historically, systemic or topical steroid therapy has been considered first-line treatments. ${ }^{12}$ Topical swallowed budesonide or fluticasone resulted in significant improvement of patient reported dysphagia and endoscopic mucosal appearance in one study. ${ }^{13}$ Isolated cases in which patients received rituximab, tacrolimus, cyclosporine, azathioprine, and mycophenolate mofetil have also been described in severe or refractory oral LP. $^{13-17}$ Systemic therapy may be warranted to prevent long-term fibrotic complications such as stenosis and strictures. Once these are formed, concurrent and repeated therapy with esophageal dilations may still be required to achieve symptomatic relief. In many cases, relapse can be expected when treatment is reduced or discontinued, resulting in continued interval therapy.

Importantly, due to chronic inflammation, ELP may have premalignant potential, as there have been multiple described cases of esophageal squamous cell carcinoma in those with ELP. The precise risk of malignant transformation of ELP is currently unknown. However, as demonstrated in a recent retrospective study of 132 patients with ELP, there is a markedly increased risk of esophageal squamous cell carcinoma (6.1\%). Given these findings and risk of malignancy, accurate and timely diagnosis is critical in these patients with close endoscopic surveillance, especially in the setting of aggravated symptoms. ${ }^{17,18}$

\section{CONFLICT OF INTEREST}

No potential conflict of interest relevant to this article was reported.

\section{ORCID}

Mahmoud Y Madi (D) https://orcid.org/0000-0001-5759-3386

\section{REFERENCES}

1. Sharma A, Białynicki-birula R, Schwartz RA, Janniger CK. Lichen planus: an update and review. Cutis 2012;90:17-23.

2. Franco DL, Islam SR, Lam-himlin DM, Fleischer DE, Pasha SF. Presentation, diagnosis, and management of esophageal lichen planus: a series of six cases. Case Rep Gastroenterol 2015;9: 253-260.

3. Fox LP, Lightdale CJ, Grossman ME. Lichen planus of the esophagus: what dermatologists need to know. J Am Acad Dermatol 2011;65:175-183.

4. Chandan VS, Murray JA, Abraham SC. Esophageal lichen planus. Arch Pathol Lab Med 2008;132:1026-1029.

5. Kern JS, Technau-Hafsi K, Schwacha H, et al. Esophageal involvement is frequent in lichen planus: study in 32 patients with 
suggestion of clinicopathologic diagnostic criteria and therapeutic implications. Eur J Gastroenterol Hepatol 2016;28: 1374-1382.

6. Katzka DA, Smyrk TC, Bruce AJ, Romero Y, Alexander JA, Murray JA. Variations in presentations of esophageal involvement in lichen planus. Clin Gastroenterol Hepatol 2010;8: 777-782.

7. Nielsen JA, Law RM, Fiman KH, Roberts CA. Esophageal lichen planus: a case report and review of the literature. World J Gastroenterol 2013;19:2278-2281.

8. Behrens A. Endoscopic imaging of esophageal lichen planus. Video GIE 2013;1:31-32.

9. Akhondi H. Sloughing esophagitis: a not so common entity. Int J Biomed Sci 2014;10:282-286.

10. Rao B, Gulati A, Jobe B, Thakkar S. Esophageal lichen planus: understanding a potentially severe stricturing disease. Case Rep Gastrointest Med 2017;2017:5480562.

11. Sato Y, Takenaka R, Matsumi A, et al. A Japanese case of esophageal lichen planus that was successfully treated with systemic corticosteroids. Intern Med 2018;57:25-29.

12. Podboy A, Sunjaya D, Smyrk TC, et al. Oesophageal lichen planus: the efficacy of topical steroid-based therapies. Aliment
Pharmacol Ther 2017;45:310-318.

13. Parmentier L, Bron BA, Prins C, Samson J, Masouyé I, Borradori L. Mucocutaneous lichen planus with esophageal involvement: successful treatment with an anti-CD20 monoclonal antibody. Arch Dermatol 2008;144:1427-1430.

14. Keate RF, Williams JW, Connolly SM. Lichen planus esophagitis: report of three patients treated with oral tacrolimus or intraesophageal corticosteroid injections or both. Dis Esophagus 2003;16:47-53.

15. Chaklader M, Morris-Larkin C, Gulliver W, McGrath J. Cyclosporine in the management of esophageal lichen planus. Can J Gastroenterol 2009;23:686-688.

16. Lear JT, English JS. Erosive and generalized lichen planus responsive to azathioprine. Clin Exp Dermatol 1996;21:56-57.

17. Frieling U, Bonsmann G, Schwarz T, Luger TA, Beissert S. Treatment of severe lichen planus with mycophenolate mofetil. J Am Acad Dermatol 2003;49:1063-1066.

18. Ravi K, Codipilly DC, Sunjaya D, Fang H, Arora AS, Katzka DA. Esophageal lichen planus is associated with a significant increase in risk of squamous cell carcinoma. Clin Gastroenterol Hepatol 2019;17:1902-1903.e1. 